\title{
STUDY OF VARIOUS SCHEMES FOR LINK RECOVERY IN WIRELESS MESH NETWORK
}

\author{
Kalyani Pendke ${ }^{1}$ and S.U.Nimbhorkar ${ }^{2}$ \\ ${ }^{1}$ Wireless Communication \& Computing, Department of Computer Science and \\ Engineering, G.H.Raisoni College Of Engneering, Nagpur \\ ${ }^{1}$ pendke@gmail.com \\ ${ }^{2}$ Department of Computer Science and Engineering, G.H.Raisoni College Of Engneering \\ , Nagpur \\ ${ }^{2}$ sonaliridhorkaregmail.com
}

\begin{abstract}
As there is a growing need for the cost effective and highly dynamic large-bandwidth networks over large coverage area, the Wireless Mesh Network provide first step towards effective communication. A Wireless Mesh Network is one of the most advanced wireless network used for communication. During their operating period, the wireless mesh network may suffer from frequent link failure which results in poor performance of network. Link failure detection plays crucial role in performance of WMN. The proposed paper presents the review of various techniques used for detection of link failure and the techniques used for recovery of wireless mesh network.
\end{abstract}

\section{KEYWORDS}

Wireless Mesh Networks (WMN); IEEE 802.16, Multipath Routing Protocol; CRWMN (Cognitive Radio based wireless mesh networks), False alert

\section{INTRODUCTION}

Wireless Mesh Network(WMN) is one of the latest wireless communication technology. Wireless Mesh Network uses multiple radio \& multiple channel architecture while previous wireless networks use single radio, single frequency architecture. By making use of multiple radio \& multiple channel architecture, WMN removes most of the problems associated with single radio \& single channel architecture in conventional system [1].

The main feature of Wireless Mesh Network is the ability of network to sustain the performance in the presence of large number of errors. In wired network a small amount of error can stops the working of entire network. But in WMN the single node is connected to multiple nodes, due to which the alternate path are always available in case of failure. The advantage of WMN is it's expandability. The new nodes can be added easily in network.

There are two types of Wireless Mesh Network :Indoor Wireless Mesh Network, Outdoor Wireless Mesh Network.

DOI : $10.5121 /$ ijans.2012.2405 
International Journal on AdHoc Networking Systems (IJANS) Vol. 2, No. 4, October 2012

1)Indoor Wireless Mesh Network :Recently Indoor wireless mesh networks become very popular as an inexpensive way to interconnect small enterprise networks. Connectivity provided by Indoor Wireless Mesh Network is of poor quality. Difficulty occurs while using the Indoor Wireless Mesh Network with traditional WiFi network. The Indoor Wireless Mesh Network should make use of TV bands rather to use WiFi ISM band. Due to low carrier frequency and improved propagation, they can implement advanced signal processing and antenna design techniques to cancel the self-interference and achieve full-duplex communication in a single band in realistic indoor scenarios.

2)Outdoor Wireless Mesh Network : Working of Outdoor Wireless mesh network is different from that of the Indoor Wireless mesh network. Outdoor Wireless mesh network is used to cover large physical region. It is widely used in large cities. Mobile Internet Access, Residential \& small Business Internet Access are some of the applications of Outdoor Wireless Mesh Network.

Rest of the papers are organized as follows, Section II describe the related work regarding link recovery in WMN. Section III describe the methods for link failure detection. Section IV describe the multipath routing in WMN. Section V describe the link recovery in Cognitive Wireless Mesh Network. Section VI conclude the paper.

\section{RELATED WORK}

\subsection{Techniques for Link recovery in WMN}

Link Recovery plays very important role in Wireless Mesh Network . The performance of Wireless Mesh Network degrades largely if there is link failure. There are various techniques used for Link Recovery in Wireless Mesh Network [2] . Some of them are listed below:

2.1.1 Initial Resource Allocation Method : In this type of algorithm, the initial planning is done for resources of the network. They use some theoretical guidelines for allocation of resources. This technique has drawback of "Global reconfiguration Changes". For small changes done for link recovery, this technique performs reconfiguration of the entire network which is not feasible in case of frequent link failure. The authors Bhati, Li \& Alcherry[3] suggested the new method namely "Joint Channel Assignment Method". For handling the channel assignment and routing problem this method make use of concepts in mathematics. The various issues like interference and channel availability are being considered in this method.

2.1.2 Greedy Channel assignment Method : This method removes the main drawback of "Initial Resource Allocation Method" i.e. reconfiguration which occurs globally [4]. In this method, the setting of only faulty link is changed rather to change entire network. The drawback of this method is "ripple effect". In this kind of effect whenever one local change occurs then it causes triggering of change to some other kind of network settings.

2.1.3 Fault Tolerant Routing Protocol : Routing protocols plays very important role in case of recovery of link failure. Whenever the link failure occurs, then to overcome that link failure we 
can route the packet via different link. For this some fault tolerant routing protocols like local rerouting or multipath routing can be used.

2.1.4 Autonomous Reconfiguration System(ARS) : A new recovery technique for wireless mesh network namely ARS is proposed by Kim and Shin[5] . This technique improves the performance of wireless mesh network largely as compared to other types of link recovery techniques. ARS generates set of reconfiguration plans by considering the range of channel \& radios of network. The feasible plan is chosen out of the set of this reconfiguration plan which maximizes the network throughput \& also satisfy some QoS constraint of network. The main drawback of ARS is that it is not cost aware reconfiguration technique.

\subsection{Need for Self- Reconfigurability :}

The link in WMN plays very important role in working of WMN. Because of link failure, the wireless mesh network is not able to perform nicely. The hand-operated network management system can be used to perform link recovery. But it is very much expensive process \& also very difficult to implement in case of dynamic link failure [6] .To overcome all the above problem there is large need for self - reconfigrability of network.

Some of the examples in which the self- reconfigurability is necessary are given as follows:

2.2.1 Recovering From Low Quality Link: In WMN the links between nodes of network may suffer interference from near by wireless network in particular region. To solve this problem , the concept of channel switch is used. In channel switch the tuned channel of link is switched to other interference free channel. In this way local link can be recovered from link failure.

2.2.2.Satisfying QoS Requirement :Sometimes it is difficult to satisfy the QoS requirement of end user.The scenario where such QoS requirement occurs are the links around conference room. This link has to deliver large amount of data in short period of time. In order to provide such QoS requirement of end user the concept of radio switch is used. In this concept the heavily loaded radios are re associated with less loaded radios.

2.2.3 Priority Use of Channel: Spectrum regulation plays an important role in working of WMNs. The predefined rules for use of spectrum may not allow some links to use certain channel in given area. Such Channels are used for handling emergency condition (e.g. Channel near hospital , public safety etc.)

\section{DETECTION OF LINK FAILURER IN WMN}

Detection of link failure in WMN is an important issue in working of WMN. There are various techniques used for detection of link failure such as cross layer model which perform timely detection of failed link. Previously routing protocol's neighbor discovery mechanisms is used for detection of link failure in WMN [7]. 
International Journal on AdHoc Networking Systems (IJANS) Vol. 2, No. 4, October 2012

3.1 Link Failure Detection using Neighbor Discovery Mechanism : In Neighbor Discovery Mechanism the concept of HELLO message is used. In this, all the nodes of network send HELLO message to all other neighbored nodes in it's communication range. After receiving number of HELLO message on the link, the link is assumed to be in good condition \& can be used for routing of packets. In the absence of HELLO messages on link for specific time interval , it is assumed that the link failure has occurred. Real Time application can not use this approach for link failure detection because large amount of delay occurs in this approach.

3.2 Link Failure Detection using Cross Layer Approach : In Cross layer Approach , whenever a MAC frame is received then the acknowledgement is sent for that frame. If the acknowledgement is not received then that frame is retransmitted. The number of times frame retransmission is performed $\&$ after that the delivery is assumed to be failed and frame is lost. Detection of link failure is performed by taking into account the number of failed delivery occurred in MAC layer along with the information about transmission errors. The advantage of cross layer approach over simple approach of neighbor discovery is the fast finding of link failure.

To ensure the correctness of link failure detection types of transmission error must be identified correctly. Basically there're are two types of transmission errors transient error and permanent error. Transient error can be removed by performing the retransmission of frames in MAC layer while the permanent errors has to handle differently. If the link is permanently failed then this type of failure is handled at routing layer by choosing alternate path for routing of packets during communication. The type of error must be detected correctly otherwise false alert occurs. False alert means the transient errors are mis-understood as permanent error. Because of this, the additional overhead of re-routing of packets is occurred. For better performance of wireless mesh network the false alert must be avoided.

\section{MULTIPATH ROUTING IN WMN}

The concept of multipath routing in important in Wireless Mesh Network . The performance of Wireless mesh network is improved significantly by using concept of multipath routing. The main objective of multipath routing is to provide fault tolerance to the network and to reduce the routing overhead . Reliability of network and load balancing of network can also be increased largely by using multipath routing. The main issue in multipath routing are the gathering of large number of control messages during routing and congestion occurring in gateway.

4.1 Flooding of Control Messages During Routing : In on demand routing the connection is established with the help of Route Request message. All the intermediate node respond to this message. All such control messages are then passed to the gateway in large amount. This results in performance degradation of entire network.

4.2 Congestion Occurring at Gateway : The gateway has to relay data packets as well as control packets. When congestion of control packets occurs at gateway then most of its time are wasted in processing of control packets. Due to this the performance of network degrades severely. 
International Journal on AdHoc Networking Systems (IJANS) Vol. 2, No. 4, October 2012

K.Valarmathi and N. Malmurugan [8] suggested new multipath routing protocol for WMN. In this protocol initially they determine interference of each link and calculate the load carried out by each link of the network. By considering this two parameter, the combined routing metric is determined. The next step is to determine multiple shortest path in the network. From the number of the shortest path, the path which has minimum value of combined metric is chosen as main path for routing. If any kind of failure occurs in main path then the transmission of packets is performed via backup path. K.Valarmathi and N. Malmurugan uses NS2(Network Simulator 2) to simulate the Multipath Routing for Improving reliability (MRIR) Protocol. They compare MRIR protocol with Multi-Path Parallel Routing Protocol(MPRP). The performance evaluation is done by considering parameters like Average End-To-End delay, Average Packet Delivery Ratio \& Overhead. This protocol shows greater performance as compared to other existing multipath routing protocol for Wireless mesh Network.

\section{LINK RECOVERY IN CRWMN}

Cognitive Radio Wireless Mesh Network (CRWMN) is new technology in Wireless Mesh Network. It is specially design to overcome the disadvantage of Single radio based Wireless Mesh Network. In CRWMN , the Cognitive radio nodes use the unutilized channels in the network without disturbing the primary user of that channel which originally have the access to the channel. After the activation of main user of the channel, the CR nodes has to release the operating channel allowing main user to use it's channel. The reassignment of channel to the affected links is performed by Cognitive Radio node.

In CRWMN , the concept of JCAR- update is used. JCAR stands for Joint Channel Assignment and Routing Update. Assignment of Channels and routing of data packets are two things which are interrelated with each other. Because of this reason, when channel re-assignment is made then the routing of path must also be updated jointly. And this is called JCAR - update.

In CRWMN, JCAR - update causes large signaling overhead which ultimately reduces the performance of network. In the paper authors Dong Heon Lee and Wha Sook Jeon [9] suggested the technique to reduce such signaling overhead in CRWMN. They suggested recovery scheme in which two step JCAR- update is performed when the primary user tries to access the channel. In first step, the routers of mesh network tries to perform the reassignment of channel to the affected links at their level without exchanging the control information. If the recovery process at this level fail then the second level of recovery is used. During Second level of recovery process the CRWMN performs re-optimization of the assigned channel over entire network.

The author of paper [10] discuss the problem of link failure detection in congested situation. They proposed an extension of the Multi-radio ad-hoc on-demand distance vector routing protocol(AODV-MR) to detect the link failure in congested environment \& also perform the recovery of path in WMN. In this method, first the difference between the quality of current link $\&$ other available link is calculated. If it exceeds some predefined value then the recover of path is performed proactively. The threshold value is determined by considering the network status. 
International Journal on AdHoc Networking Systems (IJANS) Vol. 2, No. 4, October 2012

\section{CONCLUSION}

This paper discuss the methods Neighbor Discovery Mechanism \& Cross layer Approach for detection of Link Failure in WMN. The paper presents the survey of various link recovery techniques like Initial Resource Allocation Method, Greedy Channel Assignment method \& Autonomous Reconfiguration System(ARS) in detail. It finally discuss the use of Multipath routing for recovery of Wireless Mesh Network.

\section{REFERENCES}

[1] I. Akyildiz, X. Wang, and W. Wang, "Wireless mesh networks: A survey," Comput. Network., vol. 47, no. 4, pp. 445-487, Mar. 2005.

[2] G. Murugaboopathi , T.K.S.Rathishbabu ,R.Venkatesh , “An Analysis of Reconfiguration Approaches for Recovery in Wireless Mesh Networks ", International Journal of Computer Applications , July 2012.

[3] Alicherry M, Bhatia R, and Li L, "Joint channel assignment and routing for throughput optimization in multi-radio wireless mesh networks," in Proc. ACM MobiCom, Cologne, Germany,pp. 58-72, Aug. 2005.

[4] Raniwala A and Chiueh T, "Architecture and algorithms for an IEEE 802.11-based multi-channel wireless mesh network," in Proc. IEEE INFOCOM, Miami, FL, Vol. 3, pp. 2223-2234, Mar. 2005.

[5] Kyu-Han Kim and Kang G. Shin, IEEE/ACM TRANSACTIONS ON NETWORKING, "SelfReconfigurable Wireless Mesh Networks ”, APRIL 2011

[6] Ramakrishnan R, Dr. N. Sankar Ram, Dr. Omar A. Alheyasat, “ A Cost Aware Reconfiguration Technique for Recovery in Wireless Mesh Networks”, IEEE/ICRTIT , 2012.

[7] Timo Lindhorst, Georg Lukas, Edgar Nett, "Modeling Fast Link Failure Detection for Dependable Wireless Mesh Networks", IEEE, 2010.

[8] K. Valarmathi , N,Malmurugan , "Multi Path Routing Protocol for Improving Reliability in IEEE 802.16 Wireless Mesh Networks", IEEE , 2011.

[9] Dong Heon Lee \& Wha Sook Jeon , "Channel Assignment \& Routing with Overhead Reduction for cognitive Radio- Based wireless Mesh Networks" IEEE, 2011.

[10] Byoungheon Shin Seon Yeong Han Dongman Lee , "Dynamic Link Quality Aware Routing Protocol for Multi-Radio Wireless Mesh Networks" , 26th IEEE International Conference on Advanced Information Networking and Applications , 2012 